\title{
Malattia di Cushing e adenoma ipofisario multiclonale: Ia strana coppia
}

\author{
Irene Gagliardi ${ }^{1} \cdot$ Marta Bondanelli $^{1} \cdot$ Luca Borgatti $^{2} \cdot$ Alba Gaban $^{3} \cdot$ Maria Rosaria Ambrosio $^{1} \cdot$ Maria Chiara Zatelli $^{1}$
}

Accettato: 23 aprile 2020 / Pubblicato online: 10 novembre 2020

(c) The Author(s) 2020

Fig. 1 Ricostruzioni multi-planari coronali (a) e sagittali (b) di studio preoperatorio con Tomografia Computerizzata (TC) sellare con mdc. Il processo espansivo divarica i sifoni carotidei, impegna i seni cavernosi ed esercita una cospicua impronta sul chiasma. Enhancement sostanzialmente omogeneo
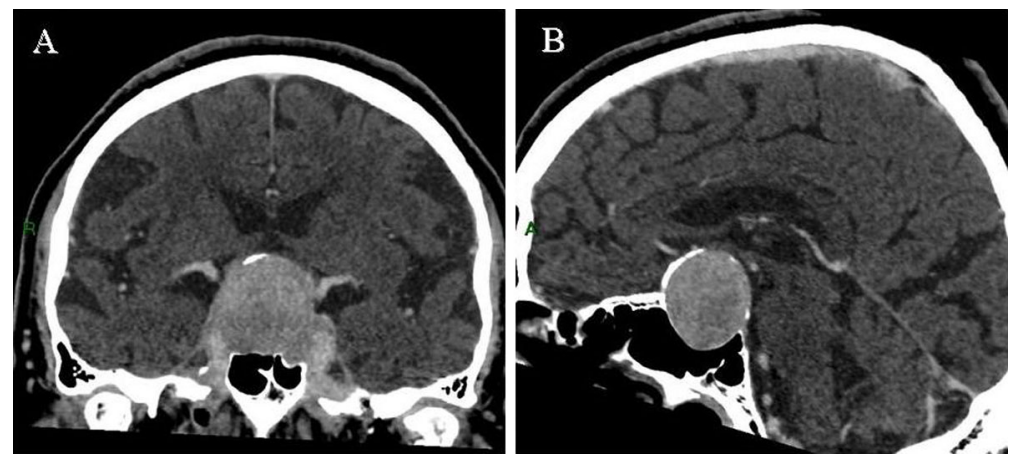

La Malattia di Cushing è una sindrome clinica da ipersecrezione di ACTH e cortisolo, perlopiù causata da un tumore neuroendocrino dell'ipofisi (PitNET). La classificazione OMS del 2017 [1] divide i PitNET in base alla secrezione ormonale e all'espressione di specifici fattori di trascrizione.

\section{Caso clinico}

Un uomo di 74 anni presenta emianopsia bitemporale e lagoftalmo sinistro paralitico. Le immagini RM e TC mostrano un espanso intra- e sopra-sellare di $44 \times 30 \times 43 \mathrm{~mm}$, che comprime le circostanti strutture nervose e vascolari (chia-

$凶$ M.R. Ambrosio

mbrmrs@unife.it

1 U.O.L. Endocrinologia, Sezione di Endocrinologia e Medicina Interna, Dipartimento di Scienze Mediche, Università degli Studi di Ferrara, Ferrara, Italia

2 U.O. di Neuroradiologia, Dipartimento di Radiologia, Azienda Ospedaliero-Universitaria di Ferrara, Ferrara, Italia

3 Unità di Anatomia Patologica, Dipartimento Oncologico-Medico Specialistico, Azienda Ospedaliero-Universitaria di Ferrara, Ferrara, Italia sma ottico, sifoni carotidei, III ventricolo, poligono di Willis, VI nervo cranico sinistro, seni cavernosi) ed erode il pavimento sellare (Fig. 1).

L'obiettività clinica è negativa per sindrome da ipersecrezione ormonale. La valutazione ormonale mostra un ipogonadismo ipogonadotropo e livelli di ACTH lievemente aumentati con cortisolo plasmatico nella norma, ma non soppressi dal test al desametasone $(1 \mathrm{mg})$.

L'esame istologico eseguito dopo rimozione chirurgica TNS dell'adenoma evidenzia due diversi cluster cellulari: uno più rappresentato $(90 \%)$ con immunoistochimica positiva per FSH/LH/ER e l'altro (10\%) positivo per ACTH e con caratteri morfologici compatibili con PitNET corticotropo sparsamente granulato. Entrambi presentano un Ki-67 $<1 \%$.

Quattro mesi dopo l'intervento la RM evidenzia la persistenza di massa tumorale coinvolgente il chiasma ottico (Fig. 2); persiste l'ipogonadismo e si evidenzia un franco ipercortisolismo ACTH-dipendente. È stata iniziata la terapia con pasireotide per l'ipercortisolismo e la terapia sostitutiva con testosterone. La ricerca di mutazioni di MEN1 è risultata negativa. 


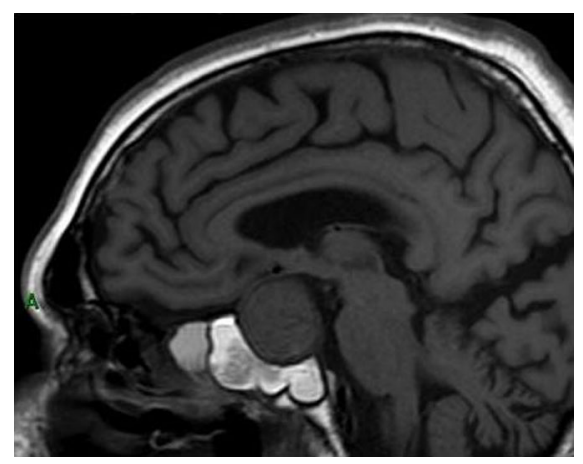

Fig. 2 Risonanza Magnetica (RM) sellare T1 Spin Echo sagittale senza mdc: controllo a 4 mesi. Riduzione dello sviluppo in senso cranio-caudale quantificabile in circa $1 \mathrm{~cm}$ con ridotta dislocazione del chiasma

Il paziente è deceduto dopo pochi mesi per complicanze postoperatorie.

\section{Conclusioni}

Il caso descrive un quadro di macroadenoma ipofisario con invasione extra-sellare composto da due diversi cluster cellulari. Ciò potrebbe spiegare il comportamento aggressivo e la necessità di una stretta sorveglianza clinica, biochimica e radiologica.

Funding Note Open access funding provided by Università degli Studi di Ferrara within the CRUI-CARE Agreement.
Conflitto di interesse Gli autori Irene Gagliardi, Marta Bondanelli, Luca Borgatti, Alba Gaban, Maria Rosaria Ambrosio e Maria Chiara Zatelli dichiarano di non avere conflitti di interesse.

Consenso informato Lo studio è stato condotto conformemente alle norme etiche stabilite dalla Dichiarazione di Helsinki del 1964.

Studi sugli animali Gli autori di questo articolo non hanno eseguito studi sugli animali.

Nota della casa editrice Springer Nature rimane neutrale in riguardo alle rivendicazioni giurisdizionali nelle mappe pubblicate e nelle affiliazioni istituzionali.

Open Access This article is licensed under a Creative Commons Attribution 4.0 International License, which permits use, sharing, adaptation, distribution and reproduction in any medium or format, as long as you give appropriate credit to the original author(s) and the source, provide a link to the Creative Commons licence, and indicate if changes were made. The images or other third party material in this article are included in the article's Creative Commons licence, unless indicated otherwise in a credit line to the material. If material is not included in the article's Creative Commons licence and your intended use is not permitted by statutory regulation or exceeds the permitted use, you will need to obtain permission directly from the copyright holder. To view a copy of this licence, visit http://creativecommons.org/licenses/by/4.0/.

\section{Bibliografia}

1. Mete O, Lopes MB (2017) Overview of the 2017 WHO classification of pituitary tumors. Endocr Pathol 28:228-243 